\title{
CAMBIO HIDRODINÁMICO Y EVOLUCIÓN DE FONDO EN RÍOS DE PLANICIE POR ESPIGONES
}

\author{
Hydridynamic change and bed evolution in lowland \\ rivers due to the presence of groynes
}

EPISTEMUS

ISSN: 2007-8196 (electrónico)

ISSN: 2007-4530 (impresa)

M.I.H. Enrique León Jiménez ${ }^{1}$

Dr. Liliana Lizárraga Mendiola ${ }^{2}$

Dr. Fabián Rivera Trejo ${ }^{3}$

M.C. Antonio Nungaray Núñez ${ }^{4}$

Ing. Jhon Díaz $\operatorname{Arcos}^{5}$

Recibido: 16 de agosto de 2016,

Aceptado: 29 de junio de 2017

Autor de Correspondencia:

M.I.H. Jesús Enrique León Jiménez

Correo:napaleon01@hotmail.com

\section{Resumen}

Existe un gran interés en la optimización de la forma, espaciado y orientación de los espigones como medidas de recuperación y protección en ríos de planicie. El objetivo principal de este estudio fue implementar una metodología numérica bidimensional, comparadas contra métodos tradicionales, que evaluaran en tres zonas la variación hidrodinámica del campo de velocidades y evolución del fondo de ríos de planicie. Con la finalidad de estimar sobre las zonas evaluadas un cambio geomorfológico. Todo esto usando un equipo Doppler ADCP y Estación total, que mediante secciones transversales estimo las geometrías complejas de las zonas evaluadas. Que al ser correlacionadas con la información granulométrica característica, evaluación numérica y métodos tradicionales. Reflejaran que actualmente en las zonas implementadas con espigones, el régimen de velocidades establecidas no supero el de velocidades permisibles, para la evolución del fondo en los ríos de planicie evaluados. No así en zonas aledañas.

Palabras clave: Espigones, Hidrodinámica, ADCP, evaluación numérica.

\begin{abstract}
There is great interest in finding the optimal shape, spacing and orientation of groynes for the purpose of recovering and protecting lowland rivers. The main objective of this study was to implement a two-dimensional numerical model for evaluating changes in the flow velocity field and river bed evolution due to the presence of groynes in lowland rivers. This methodology was compared to traditional methods by means of the estimation of geomorphological change in three different zones. The complex geometries of the study zone were estimated from cross sections obtained using ADCP equipment and a total station. These geometries were correlated with the characteristic granulometry, the numerical evaluation and the traditional methods, showing that in sites where groynes were placed, flow velocities did not surpass permissible levels, but they did in surrounding areas.
\end{abstract}

Keywords: Groynes, hydrodynamics, ADCP, numerical evaluation.

1 División Académica de Ingeniería y Arquitectura, Universidad Juárez Autónoma de Tabasco, Tabasco, México /napaleon01@hotmail.com 2 Universidad Autónoma de Hidalgo, México / lililga.Im@gmail.com

3 División Académica de Ingeniería y Arquitectura, Universidad Juárez Autónoma de Tabasco, Tabasco, México/jgfabianrivera@gmail.com

4 Consultor especializado en obras hidráulicas, Querétaro, México/jose.nungaray@icopingenieria.com.mx

5 Universidad Juárez Autónoma de Tabasco, División Académica de Ingeniería y Arquitectura, Tabasco, México / diaz.arcos.02@hotmail.com 


\section{INTRODUCCIÓN}

Los espigones son obras utilizadas mundialmente para la restauración de márgenes y zonas costeras. La optimización de la forma, espaciado y orientación de los espigones, ha generado numerosos estudios. Estas investigaciones se han enfocado al efecto hidrodinámico (campo de velocidades) que provocan estas estructuras antes de operar y después de su construcción [1,2].

La finalidad de algunos estudios es aumentar la eficiencia de operación y conocer el efecto geomorfológico negativo, causados en los ríos [3]. Ya que durante la operación de los primeros años, estas estructuras cumplen el objetivo de protección. No así durante el proceso de operación y abandono [4].

Se han realizado reproducciones de los efectos de la hidrodinámica en modelos físicos, al requerir el uso de espigones en protección de márgenes ante el efecto abrasivo de la erosión, presentado en curvas de cauces [5].

La desventaja de modelos físicos es la escasa representación por la escala. En algunos casos los aparatos de medición empleados para corroborar el efecto de la hidrodinámica del campo de velocidades se ven afectados por el propio equipo [6]. Surge interés de emigrar a nuevas técnicas de recolección de información in situ, sobre obras existentes, mediante mediciones en campo por el factor de escala, ya que éste se pierde en los modelos físicos realizados en laboratorio.

La medición en campo requiere de equipos más sofisticados para determinar el campo de velocidades en tres dimensiones; por ello, es necesaria la implementación de modelos numéricos [7]. La principal herramienta debida al desfasamiento de la medición, en escala temporal, han sido los modelos numéricos 2D y 3D, conocidos como modelos híbridos. Ya que el proceso de calibración requiere de mediciones en campo [8].

Los modelos numéricos bidimensionales proveen de una buena representación del efecto que producen los espigones al campo de velocidades en 2D. Se ha optado por seleccionar este tipo de modelos, esencialmente por el menor tiempo de cómputo simulado en su ejecución [9].

De acuerdo con las características usadas para generar modelos físicos en los laboratorios, debe controlarse la mayor cantidad de variables independientes posibles; una de ellas es el gasto líquido. Un caso propicio de estudio son los Ríos de la Sierra y el Tintillo, donde uno de los afluentes de interacción con ellos, mantiene controlada la variable de gasto líquido. Localizados en Villahermosa, Tabasco, México [10].

\section{OBJETIVO}

Implementar una metodología numérica bidimensional, comparados contra métodos tradicionales, que evalúen la variación hidrodinámica del campo de velocidades y evolución del fondo en ríos de planicie. Con la finalidad de estimar sobre las zonas evaluadas un cambio geomorfológico.

\section{PLANTEAMIENTO DEL PROBLEMA}

Actualmente no existe una comprensión clara del efec-

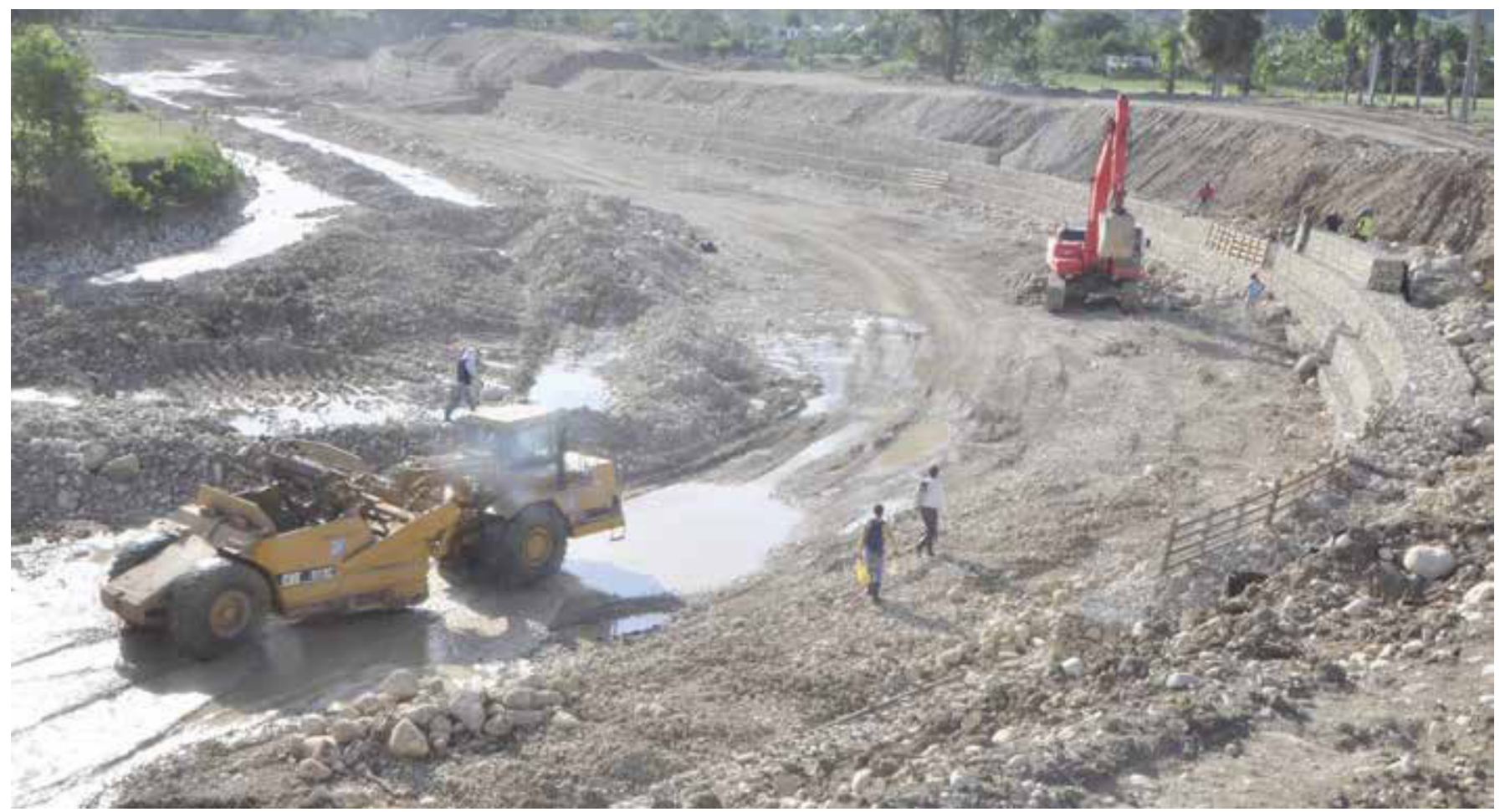




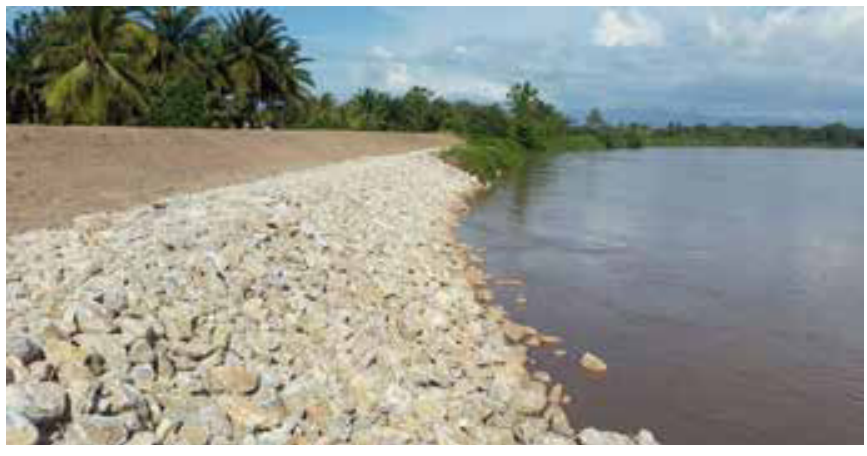

to, que causan los espigones a la hidrodinámica del campo de velocidades en cauces naturales, ya que son complejos y no tienen variables controladas. Por tal motivo, se han hecho esfuerzos por reproducir estos fenómenos en laboratorio mediante modelos físicos, concluyendo que es necesaria la medición en campo y la utilización de modelos numéricos en 2D. Generando modelos híbridos; Para reproducir situaciones reales de operación de gastos en las zonas, modelando las variables del campo de velocidad y evolución de fondo.

La zona de estudio evaluada, comprendida entre los ríos de la Sierra y el Tintillo, guardan una relación directa con el rio carrizal y esté a su vez mantiene el gasto liquido controlado en las inmediaciones de la ciudad de Villahermosa.

\section{DELIMITACIÓN DE LA ZONA DE ESTUDIO}

El río de la Sierra se localiza en las coordenadas $17^{\circ} 49^{\prime} 59.04^{\prime \prime}$ de latitud norte y $92^{\circ} 52^{\prime} 5.34^{\prime \prime}$ de longitud oeste, el río el Tintillo se localiza en las coordenadas $18^{\circ}$ $0^{\prime} 43.96^{\prime \prime}$ de latitud norte y $92^{\circ} 50^{\prime} 49.38^{\prime \prime}$ de longitud oeste [2]. (Figura 1).

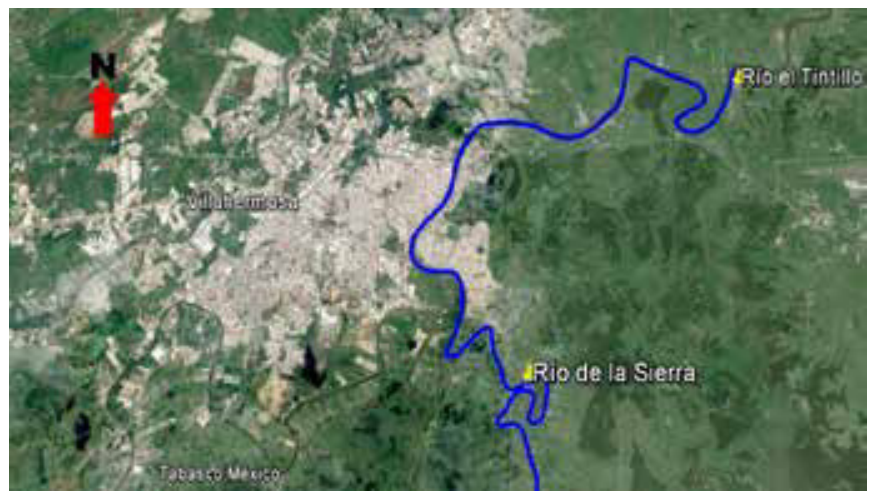

Figura 1. Localización y Ubicación de las zonas de estudio Rio tintillo y la Sierra.

\section{MEDICIONES DE CAMPO (ADCP Y ESTACIÓN TOTAL)}

Para determinar la configuración del fondo del cauce se empleó un equipo medidor acústico de efecto Doppler
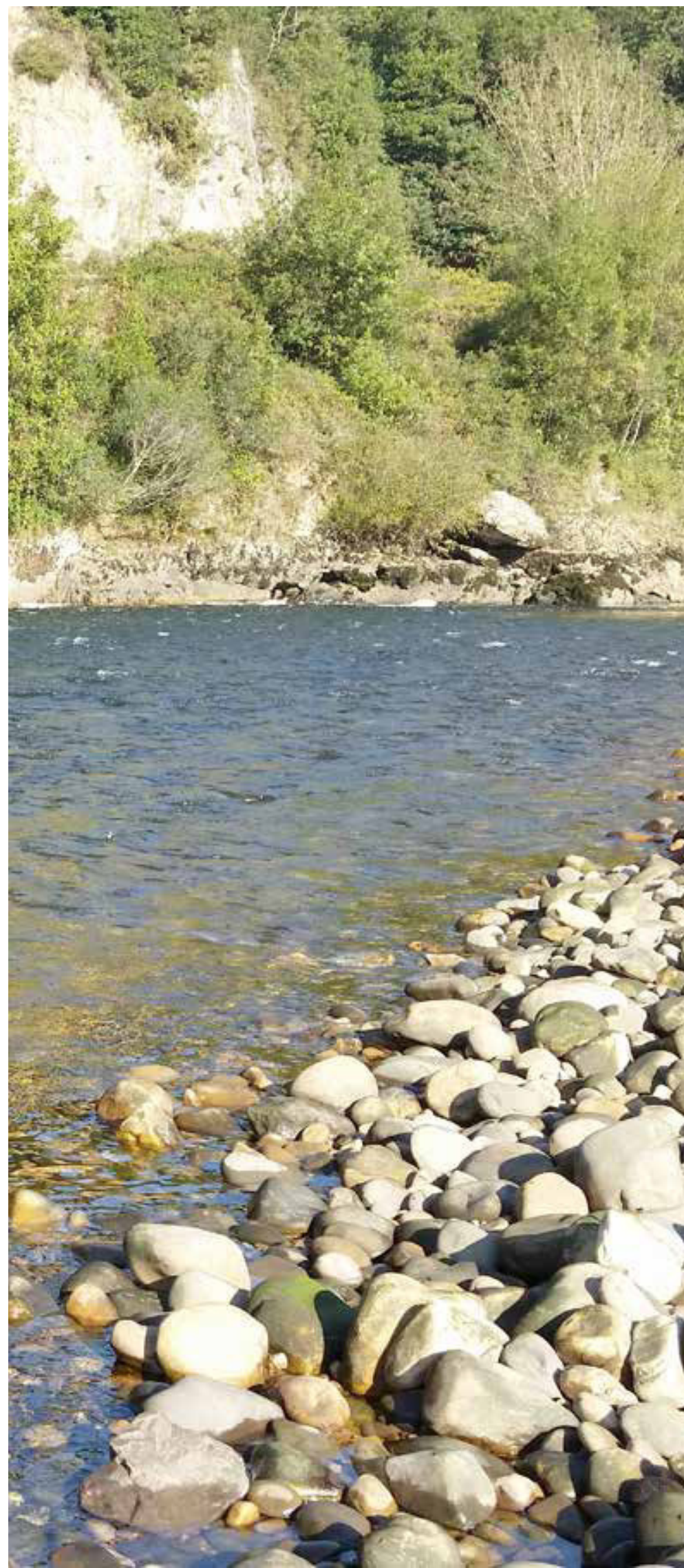


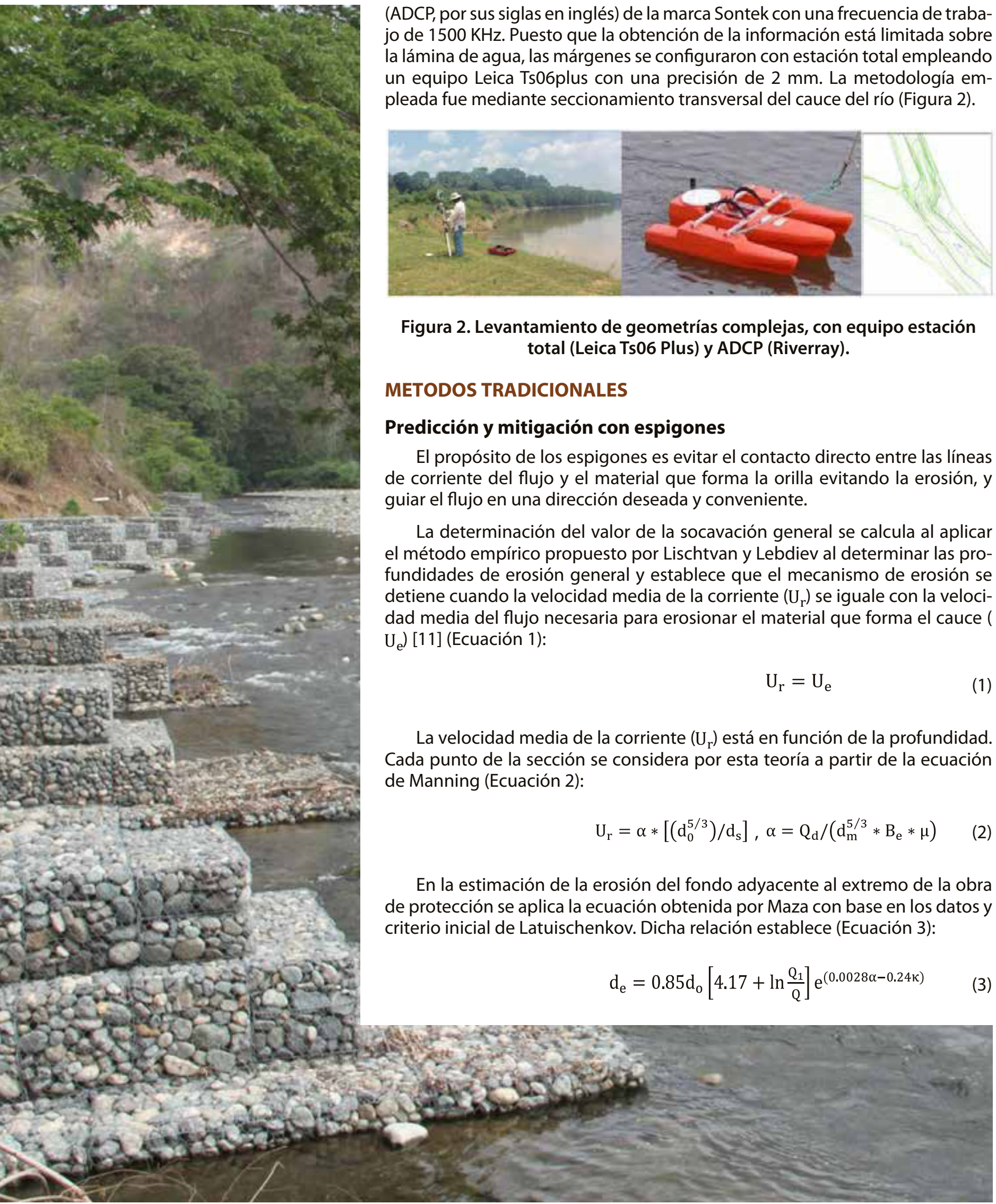


El sustento teórico de los espigones manejado en México está basado en modelos físicos realizados por Maza Alvares en laboratorios [8].

\section{Caracterización del sedimento y granulometría del río de la Sierra}

Para conocer la descripción del suelo y parámetros de predicción (socavación general y local) fue necesario tener en cuenta la granulometría (Figura 3) del río de la Sierra.

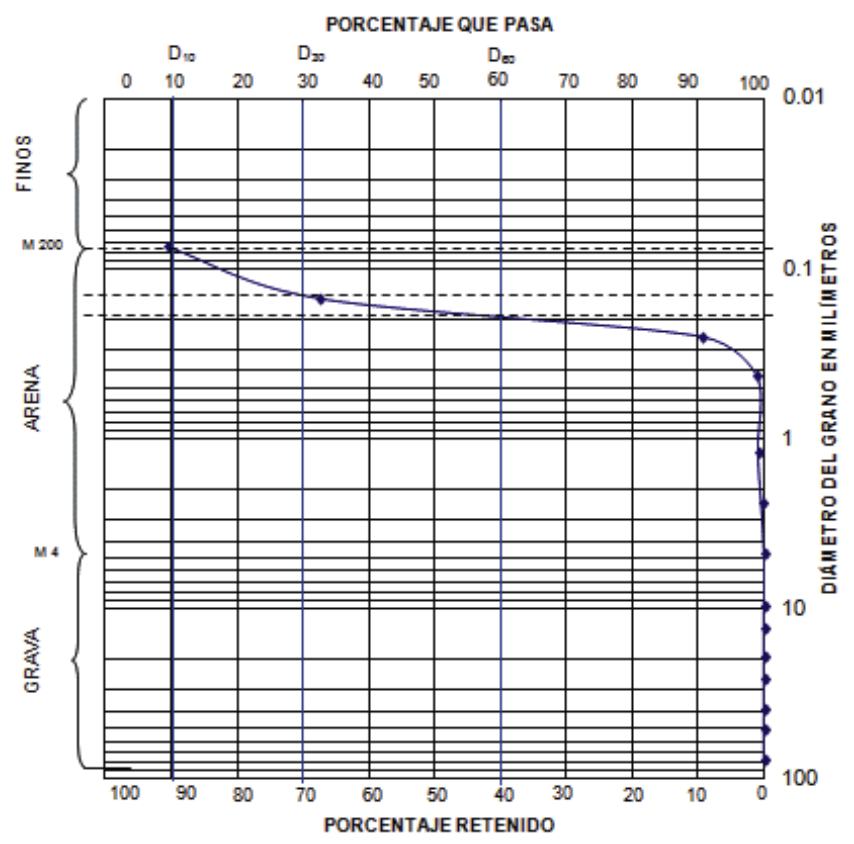

Figura 3. Análisis granulométrico del río de la Sierra.

\section{Caracterización granulométrica de la zona del río el Tintillo}

La información de la zona es imperante en la realización del estudio de velocidades permisibles, al tomar los datos del sondeo mixto realizado, dando como resultados, el peso específico de $2700 \mathrm{~kg} / \mathrm{m}^{3}$. Con un D84=0.0004 m (Figura 4).

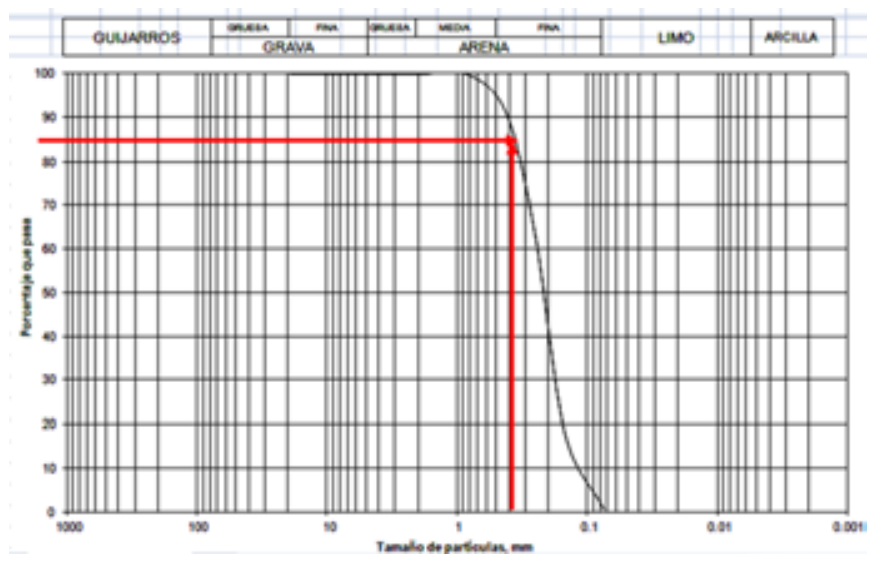

Figura 4. Análisis Granulométrico del río Tintillo.

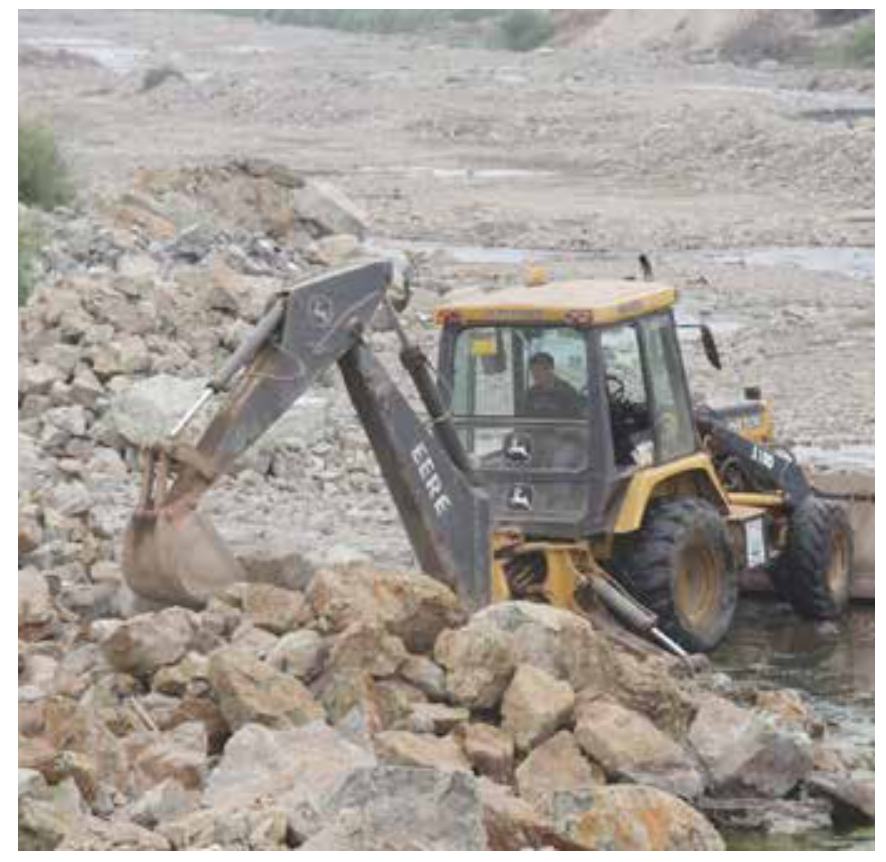

\section{SIMULACIÓN NUMÉRICA}

\section{Estimación de hidrodinámica $2 \mathrm{~d}$ en Iber 2.1.2}

El modelo Iber 2.1.2, trabaja sobre un dominio bidimensional con 3 variables definidas en cada punto del dominio: el calado $(h)$ y las dos componentes de la velocidad horizontal $\left(\mathrm{U}_{\mathrm{x}} \mathrm{y} \mathrm{U}_{\mathrm{y}}\right)$. Las ecuaciones que rigen la evolución de dichas variables se conocen como ecuaciones de aguas someras promediadas en profundidad o ecuaciones de St. Venant bidimensionales, y se expresa como (Ecuaciones 4, 5 y 6$)$ :

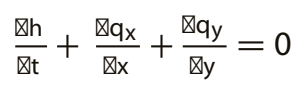

$\frac{\partial q_{x}}{\partial t}+\frac{\partial}{\partial x}\left(\frac{q_{x}^{2}}{h}+g \frac{h^{2}}{2}\right)+\frac{\partial}{\partial y}\left(\frac{q_{x} q_{y}}{h}\right)=-g h \frac{\partial z_{b}}{\partial x}-\frac{\tau_{b, x}}{\rho}+\frac{\partial}{\partial y}\left(v_{t} h \frac{\partial U_{x}}{\partial x}\right)+\frac{\partial}{\partial y}\left(v_{t} h \frac{\partial U_{x}}{\partial y}\right)(5)$

$\frac{\partial q_{y}}{\partial t}+\frac{\partial}{\partial x}\left(\frac{q_{x} q_{y}}{h}\right)+\frac{\partial}{\partial y}\left(\frac{q_{y}^{2}}{h}+g \frac{h^{2}}{2}\right)=-g h \frac{\partial z_{b}}{\partial y}-\frac{\tau_{b, y}}{\rho}+\frac{\partial}{\partial x}\left(v_{t} h \frac{\partial U_{y}}{\partial x}\right)+\frac{\partial}{\partial y}\left(v_{t} h \frac{\partial U_{y}}{\partial y}\right)(6)$

La deducción matemática de las ecuaciones de aguas poco profundas 2D se realiza promediando en profundidad las ecuaciones de Reynolds tridimensionales [12]. Concretamente, se asume una escala espacial vertical (profundidad) mucho menor que la escala horizontal, lo cual permite asumir una distribución de presión hidrostática.

La resolución de las ecuaciones de aguas someras en $2 \mathrm{D}$ emplea métodos numéricos. Es decir, en la solución numérica de las ecuaciones de flujo es necesario discretizar la malla. Las mallas discretizan las ecuaciones y se clasifican en malla estructurada y malla no estructurada (Figura 5) $[13,14]$. 


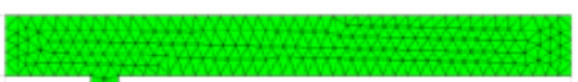

No estructurada
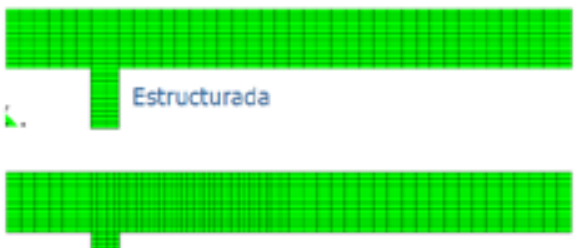

Mallado preferible

Estructurada + Refinada en confluencia

Figura 5. Estructuración del mallado en el módulo de Iber.

\section{Implementación de fondo en la modelación $2 \mathrm{~d}$ Iber 2.1.2.}

Flujo turbulento (Ecuación 7 y 8)

$\frac{\partial q_{x}}{\partial t}+\frac{\partial}{\partial x}\left(\frac{q_{x}^{2}}{h}+g \frac{h^{2}}{2}\right)+\frac{\partial}{\partial y}\left(\frac{q_{x} q_{y}}{h}\right)=-g h \frac{\partial z_{b}}{\partial x}-\frac{\tau_{b, x}}{\rho}-\frac{\partial}{\partial x}\left(h u_{x}^{\prime} u_{x}^{\prime}\right)-\frac{\partial}{\partial y}\left(h u_{x}^{\prime} u_{y}^{\prime}\right)(7)$

$\frac{\partial q_{y}}{\partial t}+\frac{\partial}{\partial x}\left(\frac{q_{x} q_{y}}{h}\right)+\frac{\partial}{\partial y}\left(\frac{q_{y}^{2}}{h}+g \frac{h^{2}}{2}\right)=-g h \frac{\partial z_{b}}{\partial y}-\frac{\tau_{b, y}}{\rho}-\frac{\partial}{\partial x}\left(h u_{x}^{\prime} u_{y}^{\prime}\right)-\frac{\partial}{\partial y}\left(h u_{y}^{\prime} u_{y}^{\prime}\right)(8)$

Modelo $k-\varepsilon$ (Ecuación 9 y 10)

$$
\frac{D k}{d t}=\frac{\partial}{\partial_{X j}}\left(\left(v+\frac{v_{t}}{\sigma_{k}}\right) \frac{\partial k}{\partial_{X j}}\right)+2 v_{t} S_{i j} S_{i j}+c_{k} \frac{u_{f}^{3}}{h}-\varepsilon
$$

$$
\frac{D \epsilon}{d t}=\frac{\partial}{\partial_{X j}}\left(\left(v+\frac{v_{t}}{\sigma_{\epsilon}}\right) \frac{\partial \varepsilon}{\partial_{X j}}\right)+c_{l \varepsilon} \frac{\varepsilon}{k} 2 v_{t} S_{i j} S_{i j}+c_{k} \frac{u_{f}^{3}}{h^{2}}-c_{2 \varepsilon} \frac{\epsilon^{2}}{k}(10)
$$

\section{Implementación de herramienta numérica Iber}

Se generó la malla del terreno natural de curvas de nivel, generando triángulos regulares al usar la técnica de error cordal [2] (figura 6).Creando una superficie NURBS (Non Uniform Rational B-Splines). Sirviéndose de la calidad de malla se justifica que entre más próximo a la unidad se encuentre, más confiable es (Figura 7). Por lo tanto representará mejores resultados y el cómputo acelera la compilación del proceso.

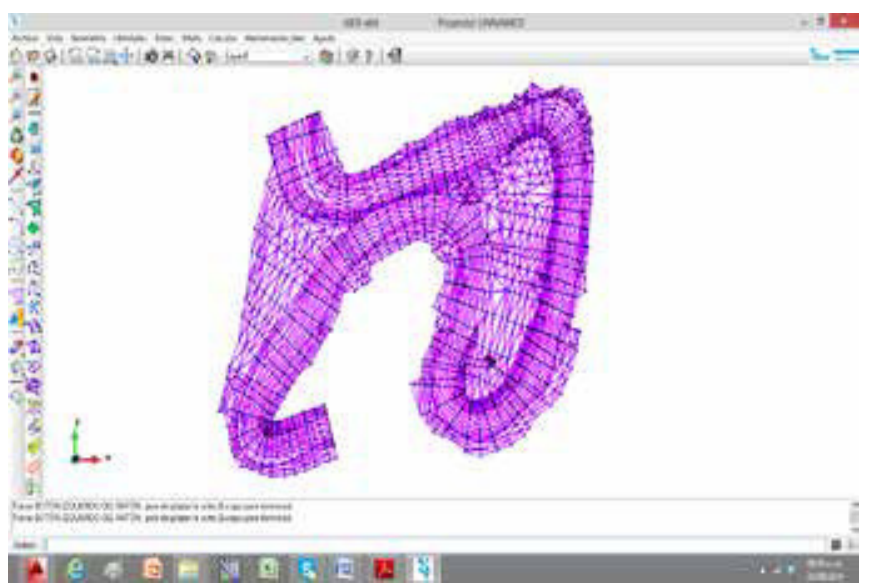

Figura 6. Superficie creada con técnica de error cordal.

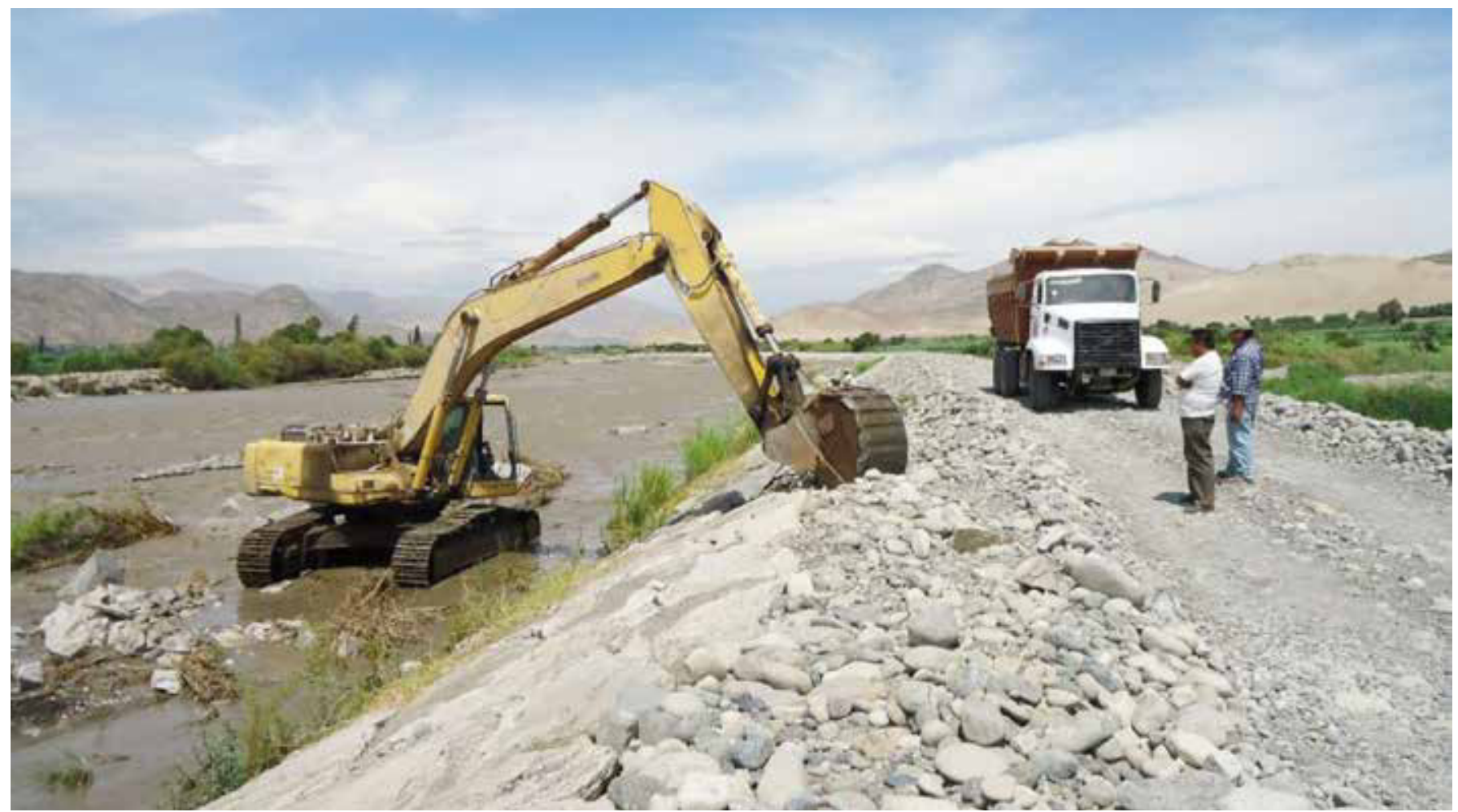




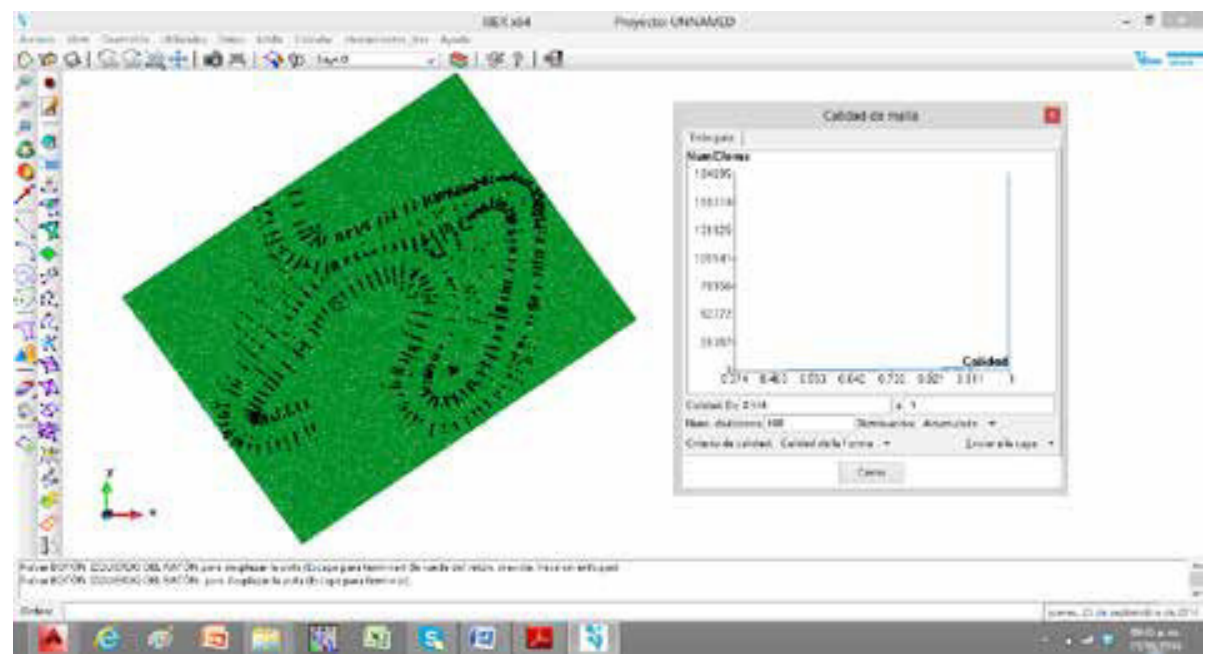

Figura 7. Mallado y calidad de la forma.

Se asignan las condiciones de contorno iniciales del modelo numérico y dentro de los parámetros asignados, se ingresa el valor del número de Manning (n), cota de agua y un gasto de entrada para un tiempo de simulación establecido y un intervalo de resultados discretizado (Figura 8).

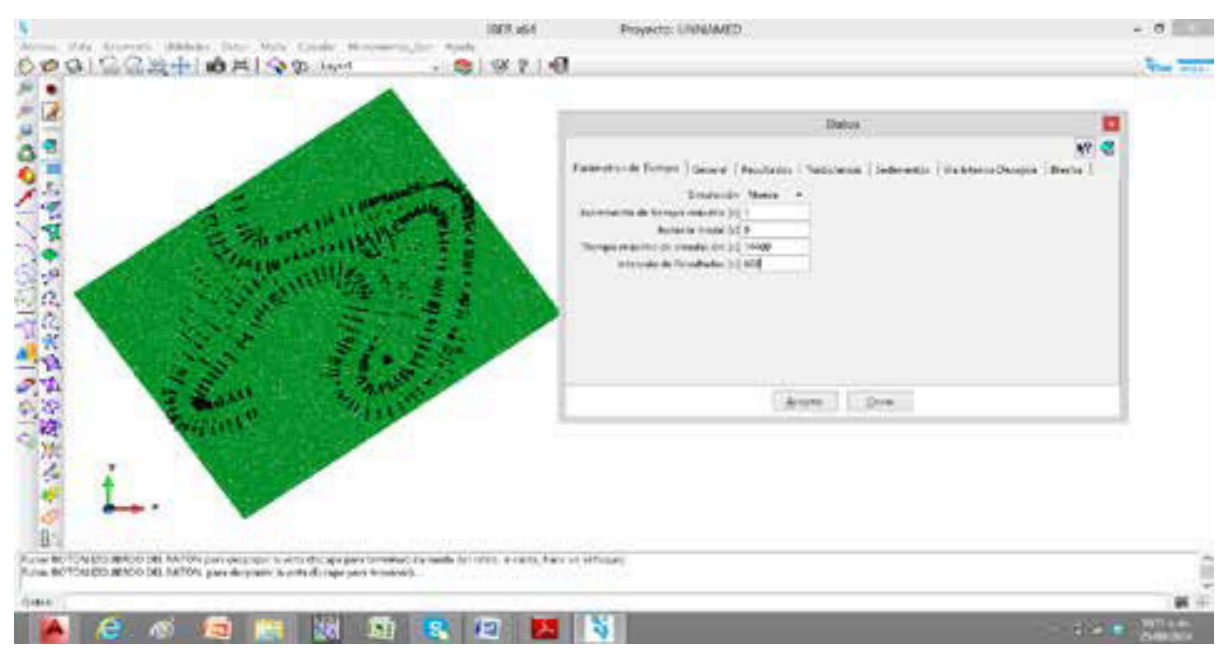

Figura 8. Asignación de valores de tiempo de simulación e intervalos de resultados.

\section{Condiciones de contorno e iniciales}

El análisis hidráulico se realizó mediante flujo permanente; que consiste en las propiedades del flujo tal como velocidad, caudal y calado en cada punto del tiempo que es constante y régimen fluvial subcritico, determinado por el número de Froude (Fr), al ser un número adimensional informa el estado del flujo y se define por la ecuación 11:

$$
\mathrm{Fr}=\frac{\mathrm{v}}{\sqrt{\mathrm{g} \cdot \mathrm{D}_{\mathrm{h}}}}=\frac{\text { Fuerzas inercia }}{\text { Fuerzas gravitatorias }}
$$

Siendo la velocidad de la sección $(\mathrm{m} / \mathrm{s}), \mathrm{g}$ la aceleración de la gravedad $\left(\mathrm{m} / \mathrm{s}^{2}\right.$ ) y $\mathrm{D}_{\mathrm{h}}$ la profundidad hidráulica $(\mathrm{m})$. De acuerdo, a esto si el número de Froude es menor a la unidad, las fuerzas gravitatorias tienen mayor importancia que las de inercia [15].

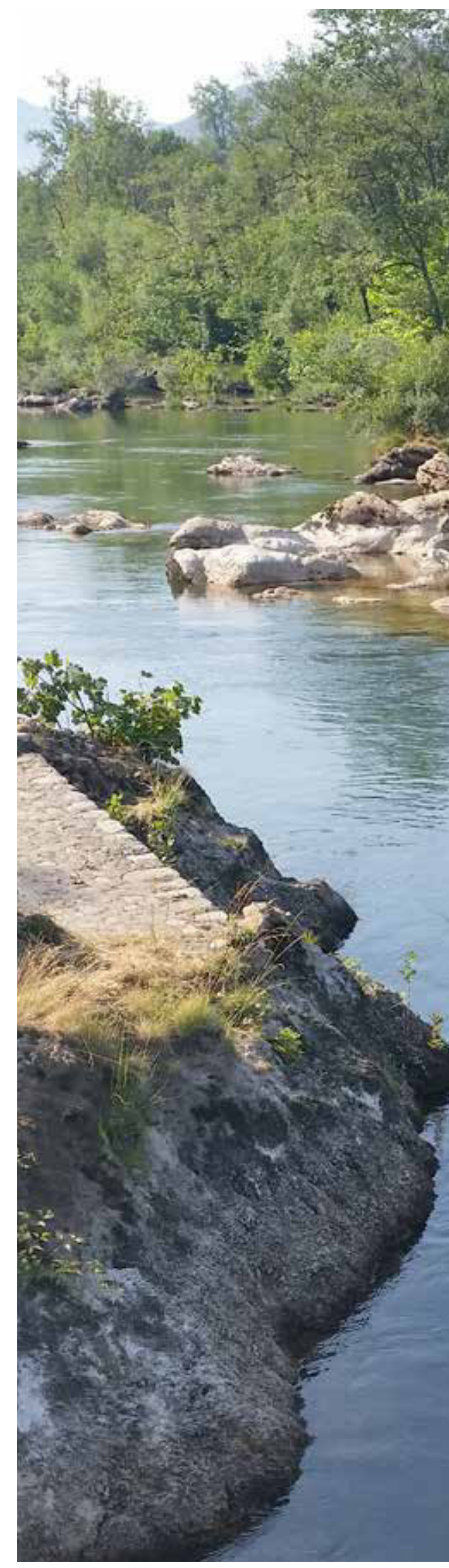


Debido a que este tipo de protecciones debe ser diseñado con el gasto máximo que transita el cauce se usaron dos criterios, el primero fue el gasto formativo, el cual es el gasto máximo que conduce el río sin desbordar hacia sus márgenes. Del manual de Estabilización y Rectificación de Ríos [11]. Para zonas donde no existe información hidrométrica. Y el segundo criterio, el gasto formativo está asociado a un periodo de retorno de 1.4 años del Manual de Gaviones [16].

Para el caso del río de la Sierra, para determinar el gasto de diseño o gasto formativo del cauce se utilizó el primer criterio, proponiendo la distribución de gastos siguientes (Tabla 1):

Tabla 1. Gatos de diseño o formativos evaluados en el rio de la sierra.

\begin{tabular}{|c|c|c|c|}
\hline Corriente & $\begin{array}{c}\text { PF1 } \\
\text { (Q m3/s) }\end{array}$ & $\begin{array}{c}\text { PF2 } \\
(Q \text { m3/s) }\end{array}$ & $\begin{array}{c}\text { PF3 } \\
\text { (Q m3/s) }\end{array}$ \\
\hline Rio sierra & 450 & 500 & 550 \\
\hline
\end{tabular}

Para el caso del rio tintillo, el modelo se alimentó con la curva gasto elevación de la sección de control aguas abajo de la escotadura Acachapan (Tabla 2), modelando todos los gastos y las elevaciones para determinar el gasto formativo, en régimen subcritico, tomando como base el segundo criterio.

Tabla 2. Gasto-elevación evaluados en el rio Tintillo.

\begin{tabular}{|c|c|}
\hline Gasto & Elevación \\
\hline 1467.01 & 2.5 \\
\hline 1757.84 & 3.37 \\
\hline 1806.03 & 3.52 \\
\hline 1922.64 & 3.87 \\
\hline 1965.88 & 4.00 \\
\hline 2142.85 & 4.61 \\
\hline 2257.15 & 4.93 \\
\hline
\end{tabular}

\section{RESULTADOS Y DISCUSIÓN}

Los resultados obtenidos de las modelaciones numéricas bidimensionales de las zonas estudiadas, que conforman los ríos de la Sierra, y el Tintillo, donde se localizan obras de protección marginal de recubrimiento a base de espigones se mencionan a continuación. Sobre la zona estudiada del rio de la Sierra, antes de la existencia de los espigones existía un régimen establecido de velocidades que oscilaba entre 0.96 y $2.4 \mathrm{~m} / \mathrm{s}$ y con espigones entre 1.23 y $3.07 \mathrm{~m} / \mathrm{s}$ (Figura 9).

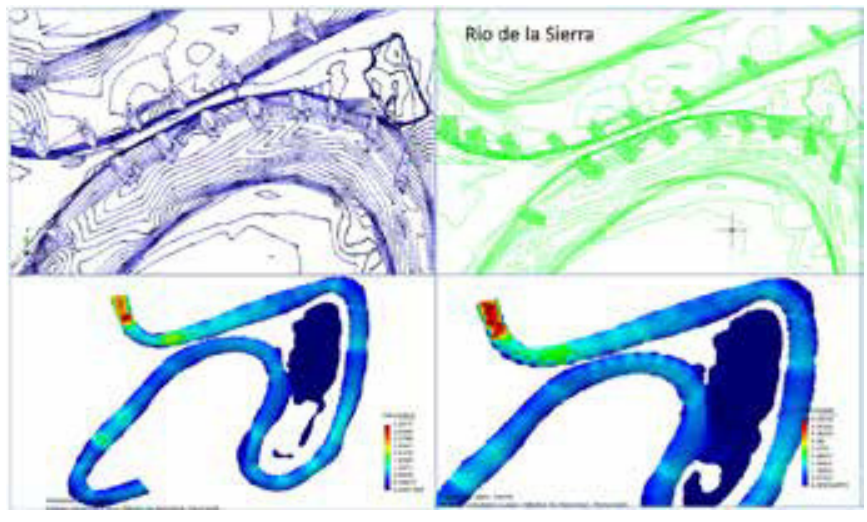

Figura 9. Cambio de régimen por acción de 16 espigones, Rio la sierra.

Este cambio de régimen está generando una evolución del fondo y márgenes, ya que el régimen admisible estimado fue de $0.91 \mathrm{~m} / \mathrm{s}$ y es superado. Con lo que actualmente la predicción de migración del fondo máxima fue $2.22 \mathrm{~m}$, erosionándose. Cuantitativamente mediante los procedimientos empíricos de laboratorio. Y una vez operando los espigones oscilaron entre 3.72 a 5.52 m, las erosiones. Demostrando que el régimen impuesto por mucho supera el admisible. Ahora bien en la zona estudiada del rio el Tintillo, antes de la existencia de los espigones había un régimen establecido de velocidades que oscilaba entre 1.19 y $1.76 \mathrm{~m} / \mathrm{s}$ y con los espigones oscilo entre 1.5 y 2.25 $\mathrm{m} / \mathrm{s}$, (figura 10).

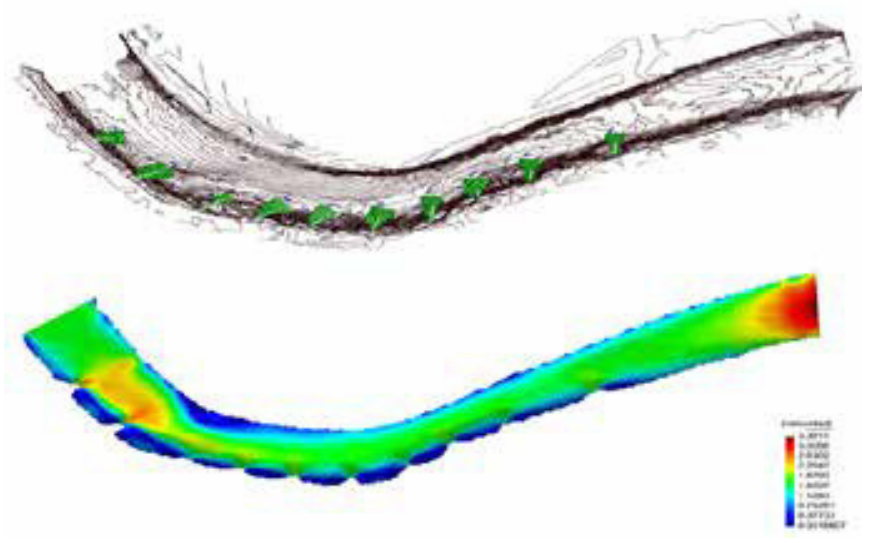

Figura 10. Cambio de régimen por acción de 10 espigones, Rio Tintillo.

Que comparado con el criterio de las ecuaciones de Shields que determina la relación de la tasa de transporte de sedimentos con la tensión de corte sobre el fondo [17], se determinó que el umbral de velocidades admisibles para suelos de arenas limo arcillosas era de $0.56 \mathrm{~m} / \mathrm{s}$, dando inicio al movimiento de las partículas cuando se presentaran velocidades superiores a esta. Estimando con ello que el promedio de socavaciones generales sobre la zona de estudio fue de $3.68 \mathrm{~m}$, y operando los espigones de 2.1 a $4.47 \mathrm{~m}$. 
Finalmente se observó que los espigones marcan una tendencia a la recuperación de márgenes, y en sitios donde existen un buen diseño y una perturbación inicial se aprecia un régimen más estable sobre el cauce y una recuperación acelerada de ella. Por esta razón, mantener un régimen estable de velocidades, hacen que el gradiente de variación de la cota de fondo respecto a su longitud sea invariante y se establezca una recuperación acelerada en ambos cauces.

\section{CONCLUSIÓN \\ Río de la Sierra}

En la zona de estudio del río de la Sierra, se obtuvieron velocidades que oscilaron entre 0.96 y $2.4 \mathrm{~m} / \mathrm{s}$. Ya operando los espigones de 1.23 a $3.07 \mathrm{~m} / \mathrm{s}$. Obteniendo con ellos socavaciones generales promedios de $2.22 \mathrm{~m}, \mathrm{Y}$ con espigones de 3.72 a $5.52 \mathrm{~m}$, en el cauce. Por lo que el régimen tanto de velocidades y de evolución de fondo se vio alterado al momento de la operación de los espigones. Cabe destacar que dicha evolución de forma natural seguiría presentándose ya que el régimen admisible estimado fue de $0.91 \mathrm{~m} / \mathrm{s}$, para el tipo de fondo de la zona de estudio, Impuesto y modelado por el régimen de gasto formativo.

\section{Río el Tintillo}

En la zona de estudio del río Tintillo, se obtuvieron velocidades que oscilaron entre 1.19 y $1.76 \mathrm{~m} / \mathrm{s}$. Ya operando los espigones de 1.5 y $2.25 \mathrm{~m} / \mathrm{s}$. Obteniendo con ellos socavaciones generales promedios de $3.68 \mathrm{~m}$. Y con espigones de 2.1 a $4.47 \mathrm{~m}$, en el cauce. Por lo que de igual forma el régimen tanto de velocidades y de evolución de fondo se vio alterado al momento de la operación de los espigones. Para este caso la evolución del fondo, de forma natural, seguiría presentándose, ya que el régimen admisible estimado fue de $0.56 \mathrm{~m} / \mathrm{s}$, en la zona de estudio, Impuesto y modelado por el régimen hidrométrico evaluado.

Puesto que durante las evaluaciones realizadas, se demostró que los espigones generan una alteración sobre las zonas aledañas donde operan. Por lo tanto deberían considerarse otras soluciones, que garanticen un efecto positivo, no acrecentando la alteración del campo de velocidades y la evolución del fondo y márgenes en cauces de planicie.

Finalmente de acuerdo a los resultados de las evaluaciones generadas, se concluye que es necesario investigar más sitios, debido a que cada sitio guarda una relación directa con la forma geomorfológica, el tipo de estratigrafía y la hidrodinámica que permite la captación de escurrimientos. Otro factor importante son los procesos antropogénicos que han provocado el aceleramiento y cambio de regímenes, tanto en la evolución del campo de velocidades y el acelerado proceso de migración de meandros (Escotaduras, Estructuras de control, Puentes).

\section{AGRADECIMIENTOS}

A la Comisión Nacional del Agua (CONAGUA) por la disponibilidad y el consentimiento del uso de la información utilizada para la elaboración de este trabajo.

\section{REFERENCIAS}

[1] M. Mccoy, G. Constantinescu and L.J. Weber, "Numerical investigation of flow hydrodynamics in a channel with a series of groynes," J. Hydraul. Eng., Vol. 134, pp.157-172, 2008.

[2] Manual de Ingeniería de Ríos. Series del Instituto de Ingeniería de la UNAM, 2012.

[3] M. Vaghefi, M. Ghodsian, and S. Neyshabouri, "Experimental study on scour around a T-shaped spur dike in a channel bend, "J. Hydraul. Eng., Vol. 138, pp. 471-474, 2012.

[4] W.H. Gregory. "Indirect Environmental Effects Of Dikes On Estuarine Tidal Channels: Thinking Outside Of The Dike For Habitat Restoration And Monitoring," J. Coast Estuaries, Vol. 27, pp. 273-282, 2004.

[5] A. Rashedipoor, A. Masjedi and R. Shojaenjad, "Investigation on scour hole around spur dike in a 180 degree flume bend, "World Appl. Sci. J., vol.19, pp. 924-928, 2012.

[6] J.G. Rivera, M.E. López, F. Uh Y O. Guillen. "Análisis de información para la identificación e inventario de los ríos y cauces en el estado de Tabasco," Universidad Juárez Autónoma de Tabasco. Mexico, Reporte de actividades, 2006.

[7] B. Ercan and B.A Younis, "Prediction of bank erosion in a reach of the Sacramento river and its mitigation with groynes," Water Resour Manage., vol. 23, pp. 3121-3147, 2009.

[8] J. Kang, H. Yeo and C. Kim, "An Experimental Study on a Characteristics of Flow around Groyne Area by Install Conditions, " J. Eng., Vol. 4, pp. 636-645, 2012.

[9] S. Abbasi S, A. Kamanbedast and J. Ahadian, "Numerical Investigation Of Angle And Geometric Of L-Shape Groin On The Flow And Erosion Regime At River Bends, "World Appl. Sci. J., vol. 15, pp. 279-284, 2011.

[10] A. N. Papanicolaou, M. Elhakeem and B. Wardman , "Calibration and verification of a $2 \mathrm{~d}$ hydrodynamic model for simulating flow around emergent bend way weir structures, "J. Hydraul. Eng., vol. 137, pp. 75-89, 2011.

[11] Manual de Estabilización y Rectificación de Ríos. Series del Instituto de Ingeniería de la UNAM, 2012.

[12] L. Cea, Puertas J. Agudo Y M.E. Vázquez, “Evaluación de corrientes litorales y frentes de marea mediante modelación bidimensional en rios y desembocaduras de ríos," Rev. Ing. Agua., vol.16, pag.13-26, 2009.

[13] E. Bladé, L. Cea, G. Corestein, E. Escolano, J. Puertas, E. Vázquez-Cendón, J. Dolz y A. Coll, " Iber: Herramienta De Simulación Numérica Del Flujo En Ríos," Rev. Int. Métodos Numér. Cálc. Diseño Ing. vol. 30, pag. 1-10, 2014.

[14] Manual Iber, Herramienta de Simulación Numérica del Flujo en Ríos. Universidad politécnica de Cataluña. 2012.

[15] Calzas Pérez Mario. (2013). Modelización del canal de la planta TEL en la U.P.T de As Pontes. Máster Tesis. Departamento de Ingeniería Matemática, Universidad Da Coruña, Coruña, España, 2013.

[16] Manual de Gaviones. Series del Instituto de Ingeniería de la UNAM, 2001.

[17] P. A. Basile, “Geomorfología e Hidráulica Fluvial Movimiento Incipiente de Sedimentos," 2003. Centro Universitario Rosario de Investigaciones HidroAmbientales. [Online]. Disponible en: http://www.fceia.unr.edu.ar/curiham/ Secciones/Publicaciones/Geomorfologia_Erosion_etc/GHF. 